Gut and Liver, Vol. 11, No. 5, September 2017, pp. 575-576

\title{
Atrophic Gastritis Increases the Risk of Gastric Cancer in Asymptomatic Population in Korea
}

\author{
Dae Young Cheung \\ Department of Internal Medicine, The Catholic University of Korea College of Medicine, Seoul, Korea
}

See "Risk Factors for Gastric Tumorigenesis in Underlying Gastric Mucosal Atrophy" by Ji Hyun Song, et al. on page 612, Vol. 11. No. 5,2017

\begin{abstract}
Atrophic gastritis is considered precursor condition for gastric cancer, but not all subjects with atrophic gastritis develop gastric cancer. In daily practice, main questions about atrophic gastritis are about that who will develop gastric cancer and when gastric cancer progress? To answer these questions, we need investigations of sufficient observation duration for enough population at risk.
\end{abstract}

Song et al. ${ }^{1}$ reported a large scale retrospective cohort about gastric tumorigenesis in subjects with atrophic gastritis. Study population was asymptomatic persons who underwent screening endoscopy for health care purpose, and the number of subject reached 10,185. Atrophic gastritis was diagnosed endoscopically in $36.5 \%$ of subjects $(3,714 / 10,185)$. Authors analyzed the risk of having atrophic gastritis by multivariate regression, and listed up risk factors including age over 55 (odds ratio [OR], 2.677; 95\% confidence interval [CI], 2.214 to $3.238 ; \mathrm{p}<0.001$ ) and male sex (OR, 2.151; 95\% CI, 1.671 to 2.770; $\mathrm{p}<0.001$ ), as well as Helicobacter pylori infection (OR, 3.224; 95\% CI, 2.089 to 4.977; in eradicated vs no infection, $\mathrm{p}<0.001$; OR, 3.405; 95\% CI, 2.779 to 4.171; in infected vs no infection, $\mathrm{p}<0.001$ ). Interesting point is that eradication of $H$. pylori infection cannot reverse the risk of atrophic change.

Gastric neoplasms including adenocarcinoma and dysplasia developed in 69 of 2,144 subjects with atrophic gastritis, in contrast to two of 3,423 without atrophic change (crude incidence for gastric neoplasm was 3.2\% and crude incidence for cancer was $1.7 \%$ for atrophic gastritis during observation period). The more extensive did the gastric atrophy present, the more probably and the more rapidly did the gastric neoplasm develop. During the follow-up duration, the incidences of gastric neoplasm was $0.1 \%$ in subjects without atrophic change, $1.6 \%$ in mild atrophic gastritis, 5.2\% in moderate atrophic gastritis, $12.0 \%$ in severe atrophic gastritis $(r=0.184, p<0.001)$. The mean time intervals to neoplasm was $6.6 \pm 2.7$ years in mild atrophic gastritis (21/1,324, 1.6\%), 5.8 \pm 3.4 years in moderate atrophic gastritis $(37 / 670,5.2 \%)$, and $4.9 \pm 2.4$ years in severe atrophic gastritis $(11 / 81,12.0 \%)(\mathrm{r}=-0.188, \mathrm{p}=0.035)$. Regarding the tumorigenic potential, study results showed escalation of risk according to the extent and duration of atrophy. And the risk of neoplasm development was significantly influenced by older age $(>55)$, alcohol consumption, presence of moderate to severe atrophic change and presence of intestinal metaplasia.

This study enlarged our knowledge about the risk size of atrophic gastritis for gastric cancer. The clinical characteristics of atrophic gastritis can vary among the population and region. $H$. pylori prevalence and dietary habit influence the pattern of atrophic gastritis. Multifocal atrophic gastritis is common in $H$. pylori high prevalent area such as East Asian countries. This type of atrophic gastritis often confined to antrum and extensive body atrophic change with high serum gastrin level is not frequent. Most patients who were endoscopically diagnosed atrophic in Korea are belong to this category. On contrarily, diffuse corporal atrophic gastritis is often occurring in European population. Patients with diffuse corporal atrophic gastritis exhibit achlorhydria or hypochlorhydria and hypergastrinemia. In a Scandinavian cohort study for 300 subjects with atrophic gastritis, during a median follow-up of 4.3 years (range, 1.0 to 16.5 years), 15 gastric neoplastic lesions were detected in 14 of the 300 patients with atrophic gastritis: three were gastric cancer, whereas 12 were noninvasive neoplasia. The annual incidence

Correspondence to: Dae Young Cheung

Department of Internal Medicine, Yeouido St. Mary’s Hospital, The Catholic University of Korea College of Medicine, 222 Banpo-daero, Seocho-gu, Seoul 06591, Korea

Tel: +82-2-3779-1519, Fax: +82-2-3779-1331, E-mail: adagio@catholic.ac.kr pISSN 1976-2283 eISSN 2005-1212 https://doi.org/10.5009/gnl17356

@ This is an Open Access article distributed under the terms of the Creative Commons Attribution Non-Commercial License (http://creativecommons.org/licenses/by-nc/4.0) which permits unrestricted non-commercial use, distribution, and reproduction in any medium, provided the original work is properly cited. 
for gastric neoplastic lesions was 1\%. Cox regression analysis identified the following risk factors: age over 50 years (hazard ratio [HR], 8.8; 95\% CI, 1.2 to 68.4), atrophic pangastritis (HR, 4.5; $95 \%$ CI, 1.5 to 14.1 ) and severe intestinal metaplasia in the gastric body (HR, 4.0; 95\% CI, 1.3 to 11.8 ). ${ }^{2}$ In British study, a cohort of 166 high-risk patients reported that 14 cancers were detected over 10 years (8.4\%). ${ }^{3}$ Due to the hypergastrinemia, Rindi type I neuroendocrine tumor of stomach is another concern about atrophic gastritis in Scandinavian population. A study reported the annual incidence rate person-year of atrophic gastritis were $0.25 \%$ (95\% CI, $0.067 \%$ to $0.63 \%$ ), $0.43 \%$ (95\% CI, $0.17 \%$ to $0.89 \%$ ), and $0.68 \%$ (95\% CI, $0.34 \%$ to $1.21 \%$ ) for gastric cancer, dysplasia, and type I-gastric carcinoids, respectively. ${ }^{4}$ In Netherland study, 92,250 patient with precursor lesion were followed up for gastric cancer. The annual incidence of gastric cancer was $0.1 \%$ for patients with atrophic gastritis, 0.25\% for intestinal metaplasia, 0.6\% for mild-to-moderate dysplasia, and 6\% for severe dysplasia within 5 years after diagnosis. ${ }^{5}$ Age over 75 (HR, 3.75; 95\% CI, 2.8 to 5.1) and male sex (HR, $1.50 ; 95 \%$ CI, 1.3 to 1.7 ) were found to significantly increase the risk of gastric cancer.

Another issue on atrophic gastritis is about the diagnosis. The clinical presentation of atrophic gastritis is absent. Endoscopic examination and histological evaluation are believed to be the reference standard for diagnosis of atrophy. Kimura-Takemoto protocol grades the extent of atrophy according to the involved area and is commonly employed approach in practice. But endoscopic decision can be influenced by luminal air inflation and endoscopist's expertise. Authors of this study describes that their interobserver agreement was low at start, but improved with internal meetings and educations and reached just up to $\kappa$-value of 0.62 . Considering that the study needed a long-term follow-up duration, newer risk stratification systems of atrophic gastritis for gastric cancer could not be employed in this study. $\mathrm{ABC}$ method of Japan was proposed to use the pepsinogen I and II level as a serology marker in $2011^{6}$ and the Operative Link for Gastritis Assessment was proposed in $2005 .^{7}$

This study provides information about gastric cancer risk of atrophic gastritis who were diagnosed by using endoscopic examination in Korea. The crude incidence for cancer was 1.7\% for atrophic gastritis during observation period. This is comparable to the result of European studies. Of course, not all patients progress to carcinogenesis, we need to be more cautious when patients have additional risk factors including older age, alcohol consumption and extensive atrophic change or intestinal metaplasia.

\section{CONFLICTS OF INTEREST}

No potential conflict of interest relevant to this article was reported.

\section{REFERENCES}

1. Song JH, Kim SG, Jin EH, Lim JH, Yang SY. Risk factors for gastric tumorigenesis in underlying gastric mucosal atrophy. Gut Liver 2017;11:612-619.

2. Vannella L, Lahner E, Osborn J, et al. Risk factors for progression to gastric neoplastic lesions in patients with atrophic gastritis. Aliment Pharmacol Ther 2010;31:1042-1050.

3. Whiting JL, Sigurdsson A, Rowlands DC, Hallissey MT, Fielding JW. The long term results of endoscopic surveillance of premalignant gastric lesions. Gut 2002;50:378-381.

4. Lahner E, Esposito G, Pilozzi E, et al. Occurrence of gastric cancer and carcinoids in atrophic gastritis during prospective long-term follow up. Scand J Gastroenterol 2015;50:856-865.

5. de Vries AC, van Grieken NC, Looman CW, et al. Gastric cancer risk in patients with premalignant gastric lesions: a nationwide cohort study in the Netherlands. Gastroenterology 2008;134:945952.

6. Miki K. Gastric cancer screening by combined assay for serum anti-Helicobacter pylori IgG antibody and serum pepsinogen levels: “ABC method”. Proc Jpn Acad Ser B Phys Biol Sci 2011;87:405414

7. Rugge M, Genta RM. Staging and grading of chronic gastritis. Hum Pathol 2005;36:228-233. 